Paidéia, 2006, 16(35), 315-325

\title{
QUALIDADE CONJUGAL: MAPEANDO CONCEITOS ${ }^{1}$
}

\author{
Clarisse Mosmann ${ }^{2}$ \\ Adriana Wagner \\ Pontifícia Universidade Católica do Rio Grande do Sul \\ Terezinha Féres-Carneiro \\ Pontifícia Universidade Católica do Rio de Janeiro
}

\begin{abstract}
Resumo: Apesar da ampla utilização do conceito de qualidade conjugal, identifica-se falta de clareza conceitual acerca das variáveis que o compõem. Esse artigo apresenta revisão da literatura na área com o objetivo de mapear o conceito de qualidade conjugal. Foram analisadas sete principais teorias sobre o tema: Troca Social, Comportamental, Apego, Teoria da Crise, Interacionismo Simbólico. Pelos postulados propostos nas diferentes teorias, podem-se identificar três grupos de variáveis fundamentais na definição da qualidade conjugal: recursos pessoais dos cônjuges, contexto de inserção do casal e processos adaptativos. Neste sentido, a qualidade conjugal é resultado do processo dinâmico e interativo do casal, razão deste caráter multidimensional.
\end{abstract}

Palavras-chave: Satisfação Conjugal; Ajustamento Conjugal; Qualidade Conjugal.

\section{MARITAL QUALITY: MAPPING OUT CONCEPTS}

Abstract: Despite the wide use of marital quality concept, it is identified a lack of variables conceptual clarity. This article presents an area literature review with the objective of mapping out the conjugal concept. Seven theories about this subject were analysed: Social Exchange Theory, Behaviourist Theory, Attachment Theory, Crisis Theory, Simbolic Interactionism Theory, Family Systems Theory, Adaptation of Vulnerability to Stress Model. It is possible to identify three groups of variables in the definition of conjugal quality: spouses personal resources, context in which the couple is inserted, adapting processes. Hence, conjugal quality is the result of couple dynamic and interactive process, consequence of a multidimensional character.

Key words: Marital Satisfaction; Marital Adjustment; Marital Quality.

\section{Introdução}

Tradicionalmente a palavra casamento associa-se a idéias românticas, remetendo à frase popular "e viveram felizes para sempre". Entretanto, um acontecimento que é marcado inicialmente pelo encantamento e otimismo, muitas vezes, acaba não sendo tão duradouro. Para muitos, a união que se inicia como fonte de satisfação termina como de frustração. Frente a esse fenômeno tão comum, perguntase então: $\mathrm{O}$ que acontece neste percurso?

A relevância do tema baseia-se nas evidências de que a maioria das pessoas irá se casar ou experimentar algum tipo de união em determinado período

\footnotetext{
${ }^{1}$ Artigo recebido em 24/07/2006 e aceito para publicação em 15/01/2007.

2 Endereço para correspondência: Clarisse Mosmann, Praça Carlos Simão Arnt n. 105 apto. 801, CEP: 90450-110, Porto Alegre - RS, E-mail: clarisse@ redemeta.com.br
}

de suas vidas. Apesar disso, dados estatísticos norte - americanos mostram que quase a metade das primeiras uniões termina em separação ou divórcio (Bradbury, Fincham \& Beach, 2000).

Apesar deste panorama pouco entusiasmante, o casamento ainda é o objetivo de muitas pessoas; um crescente número de jovens atualmente expressa o desejo de se casar e dados recentes do IBGE mostram que em 2003 foram realizados 748.981 casamentos oficiais no Brasil; isso demonstra que mesmo com o aumento de uniões estáveis, dentre as pessoas que já viveram um, ou mais casamentos que não foram bem-sucedidos, a maioria segue buscando um relacionamento que lhes traga satisfação e felicidade (Jablonski, 1991; Falcke, Diehl \& Wagner, 2002; Féres-Carneiro, 2003). 
A partir de tais evidências, surge o questionamento: $\mathrm{O}$ que leva as pessoas a reinvestirem no casamento, mesmo tendo passado por experiências conjugais insatisfatórias? Afinal, o que define um relacionamento satisfatório?

Essas são indagações que têm estado com freqüência presentes na vida cotidiana nos dias atuais. Em conversas entre amigos, em revistas de grande circulação nacional, em programas de televisão, o tema é recorrente. Existe uma busca em entender o que determina um relacionamento conjugal bem-sucedido, e no meio acadêmico, diversas pesquisas têm sido realizadas com tais objetivos.

Apesar do desejo de encontrar fórmulas mágicas que ofereçam uma receita de matrimônio feliz, as investigações científicas feitas na área mostram que devido à complexidade do fenômeno não há respostas simples para esse questionamento (Johnson, Amoloza \& Booth, 1992; Karney \& Bradbury, 1995; Amato, Johnson, Booth \& Rogers, 2003).

Especificamente, a conceituação do que seria um casamento satisfatório é tarefa árdua não só para os leigos, mas também para o meio científico. Se se pensar que o casamento envolve dois seres humanos e a complexidade de suas vivências prévias particulares, os quais vêm a estabelecer uma vida nova, podese vislumbrar o imenso número de fatores que se interconectam na vida a dois (Karney \& Bradbury, 1995).

Ao se analisarem as pesquisas internacionais da área, na última década, identifica-se um grande número de estudos que apontam para um alto índice de fatores que se associam à definição deste conceito. Os dados mostram que a qualidade do relacionamento conjugal estaria relacionada ao bem-estar dos cônjuges e seus filhos (Erel \& Burman, 1995; Shek, 1998, 2001), às respostas fisiológicas dos cônjuges (Gottman, Coan, Carrere \& Swanson, 1998), às variáveis sóciodemográficas (Perry-Jenkins, Repetti \& Crouter, 2000; Cotton, Burton \& Rushing, 2003), à saúde física do casal (Burman \& Margolin, 1992) à depressão (Beach, Katz, Sooyeon \& Brody, 2003; Uebelacker, Courtnage \& Whisman, 2003) à psicopatologia (Whisman, 1999), às características de personalidade (Ye, We \& Wang, 1999), e à combinações entre estas variáveis (O’Leary \& Smith,
1991; Neff \& Karney, 2003; Kline \& Stafford, 2004), dentre outras.

E todas elas estariam associadas à qualidade da relação conjugal, entretanto, apesar do grande número de estudos sobre o tema, isto não significa profundidade no conhecimento derivado dos mesmos (Bradbury \& cols., 2000). Identifica-se uma tendência de ateorização em tais estudos; grande parte das pesquisas não expressa de forma clara ou não apresenta uma teoria orientadora da investigação. E, não havendo uma linha teórica comum para guiar a seleção das variáveis e dos procedimentos metodológicos, surgem tentativas de replicar estudos já realizados.

Assim, os últimos cinqüenta anos de pesquisas sobre o casamento geraram um aumento crescente das variáveis supostamente preditoras do sucesso conjugal, mas sem um aprofundamento dos resultados, o que não propiciou um avanço efetivo no entendimento dos processos conjugais (Karney \& Bradbury, 1995).

O que tem se multiplicado são muito mais perguntas que repostas. Procura-se saber porque alguns casais são estáveis e outros não, uns felizes enquanto outros infelizes, mas não há uma busca para compreender a dinâmica e desenvolver modelos explicativos dos mecanismos que geram essas distintas relações.

Ao se detectar a falta de tais modelos, identifica-se também uma dificuldade na conceituação teórica da qualidade conjugal, sendo essa lacuna conceitual atribuída pelos estudiosos à subjetividade implícita na avaliação de cada participante sobre o que considera ser satisfatório em um casamento.

Devido a essa falta de clareza conceitual percebe-se, ao avaliar a literatura recente sobre o tema, que as pesquisas sobre conjugalidade estão centradas em três conceitos básicos: a satisfação, o ajustamento e a qualidade conjugal (O'Leary \& Smith, 1991; Glenn, 1998; Gottman \& Notarius, 2002); e o problema, de acordo com os pesquisadores, é que esses conceitos passaram a ser utilizados em muitas investigações como sinônimos.

Essa lacuna se reflete também na multiplicidade de escalas criadas para mensurar a qualidade conjugal e a pouca clareza conceitual influencia na forma como a grande quantidade de instrumentos 
tem sido utilizada nas pesquisas (Fincham \& Bradbury,1987).

Neste sentido, conceituar teoricamente o que é denominado de qualidade conjugal pode promover avanço nas investigações de uma área de crescente interesse do meio científico. Ademais, o refinamento metodológico nos instrumentos de mensuração pode também aprimorar o trabalho clínico dos terapeutas no tratamento do número cada vez maior de casais que procuram ajuda para lidar com suas dificuldades conjugais.

Assim, o presente artigo tem como objetivo revisar a literatura da área na tentativa de mapear os aspectos relacionados ao conceito de qualidade conjugal.

\section{Perspectivas teóricas da qualidade conjugal}

A qualidade conjugal é um construto com uma longa e controversa história. A primeira medida de qualidade conjugal foi realizada por Terman, Buttenwieser, Fergunson e Wilson em 1938 (conforme citado por Heyman, Sayers \& Bellack, 1994) que utilizou apenas uma pergunta para avaliar a felicidade"satisfação dos cônjuges: "O que basicamente diferencia os casais felizes dos infelizes?"

Essa pesquisa teve o mérito de ser pioneira na tentativa de apresentar respostas ao fenômeno, entretanto, esse questionamento explicita a subjetividade da questão e a falta de perspectiva teórica dos pesquisadores ao acreditarem que apenas uma pergunta poderia dar conta do fenômeno (Gottman \& Notarius, 2002).

Seis décadas depois, muitos pesquisadores ainda não apresentam clareza teórica em seus estudos sobre conjugalidade. Entretanto, existem quatro teorias que influenciaram consideravelmente as investigações da área.

A Social Exchange Theory é a mais citada nas pesquisas sobre qualidade conjugal; ela é derivada da teoria de Thibaut e Kelley (1959) sobre a interdependência, a qual postula que num relacionamento os acontecimentos vividos pelos cônjuges resultam de sua interação com o meio em que estão inseridos, o que gera uma sucessão de desafios que exigem adaptações dos membros do casal, tanto en- tre si como no relacionamento com os que os rodeiam.

Nas décadas de sessenta e setenta, Levinger $(1965,1976)$ foi o primeiro a aplicar esses conceitos ao casamento. Para ele o sucesso do matrimônio relaciona-se à capacidade dos cônjuges de comparar os aspectos satisfatórios como a segurança emocional, a realização sexual e a formação de uma família, com os que podem ser desafiadores como problemas financeiros, preconceitos sociais e religiosos, entre outros. De acordo com esta teoria as uniões terminam quando uma conjunção de fatores combina mais desafios e insatisfações que aspectos satisfatórios, poucos impedimentos para separação e muitas alternativas atrativas fora do matrimonio.

Posteriormente, utilizando essas idéias, Lewis e Spanier $(1979,1982)$ formaram uma tipologia dos relacionamentos conjugais em que a satisfação e a estabilidade são concebidas como dimensões ortogonais. A combinação entre as dimensões é expressa no quadro abaixo:

Quadro 1: Tipologia de Lewis e Spanier $(1979,1982)$

\begin{tabular}{|l|l|l|}
\hline \multirow{4}{*}{ Tipos de Casais } & Grau de Satisfacão & Grau de Estabolididade \\
\cline { 2 - 3 } & Alto & Alto \\
\cline { 2 - 3 } & Alto & Baixo \\
\cline { 2 - 3 } & Baixo & Alto \\
\cline { 2 - 3 } & Baixo & Baixo \\
\hline
\end{tabular}

Os conceitos dessa tipologia explicam que casais insatisfeitos, mas estáveis, por exemplo, são aqueles em que as atrações e aspectos satisfatórios derivados do relacionamento são poucos, mas os obstáculos à separação muitos.

Essa teoria possui como ponto forte considerar, tanto como atrações como obstáculos ao casamento, todos os contextos em que se insere o casal. Assim, numa aproximação à perspectiva ecológica (Brofenbrenner, 1996) considera-se o micro e o macro contextos, incluindo-se no entendimento a interdependências das variáveis envolvidas no fenômeno. Talvez esse seja o motivo de um grande número de pesquisas ter sido realizado a partir desta orientação teórica (O'Leary \& Smith, 1991; Johnson \& cols., 1992; Heyman \& cols., 1994).

O conceito de ajustamento conjugal desenvolvido por Spanier e Cole (1976), mesmo anterior à 
tipologia criada por Lewis e Spanier (1979;1982) é um dos mais utilizados até hoje nas pesquisas sobre conjugalidade. Especificamente, o Diadic Adjustment Scale - DAS, elaborado a partir de tais conceitos e dimensões, serviu como instrumento de medida para mais de 1000 investigações. De acordo com Spanier e Cole (1976) o ajustamento conjugal reflete os processos conjugais, a comunicação e os resultados destes, que seriam o nível de ajustamento do casal.

Muitas críticas são feitas ao conceito de ajustamento e, em especial, ao DAS. De acordo com Norton (1983) a dificuldade metodológica do DAS é que a escala combina processos interacionais (desentendimentos, compartilhar idéias, entre outros) com resultados (avaliações subjetivas do nível de felicidade do casal). Para o autor, essa combinação gera resultados que devem ser interpretados com cautela uma vez que as correlações entre as variáveis independentes - estilos de comunicação e satisfação - aumentam porque a primeira (comunicação) já está incluída na definição de satisfação conjugal da escala.

Neste caso específico, a crítica ao conceito de ajustamento conjugal reside na impossibilidade de verificar exatamente em que ele se distingue da satisfação conjugal (Heyman \& cols., 1994).

Neste sentido deve-se retornar à teoria que embasa o conceito. Na Teoria da Troca Social -, como já colocado, o ajustamento conjugal é resultado da interação entre os processos sociais e os desafios que ele promove para o casal. A satisfação e o ajustamento são vistos como complementares já que se consideram satisfeitos os casais de acordo com a quantidade de obstáculos e recompensas que têm do meio, e, em consequiência, como se ajustam a eles. Entretanto, essas conceituações são baseadas em percepções e não analisam os comportamentos dos cônjuges frente aos desafios (Gottman, 1982).

Posteriormente, a teoria que veio a desenvolver a análise dos comportamentos dos casais frente às dificuldades também é derivada do trabalho de Thibaut e Kelley (1959) e provocou grande desenvolvimento nas pesquisas de laboratório sobre a conjugalidade. A Behavioral Theory focalizou seus esforços nas ações dos cônjuges para resolução de seus problemas.
Essa teoria tem como premissa que os comportamentos positivos dos cônjuges aumentam sua avaliação positiva do casamento e, por consequiência, seu nível de satisfação. Assim, comportamentos considerados negativos pelo outro gerariam avaliações negativas do matrimônio. Esse postulado embasou o desenvolvimento do conceito das atribuições que os cônjuges têm sobre os comportamentos do parceiro.

A Teoria Comportamental sugere que as respostas cognitivas aos comportamentos do outro afetam o matrimônio e com o tempo a acumulação dessas experiências influencia gradualmente, positiva ou negativamente, na avaliação e na conseqüente satisfação que os cônjuges experimentam (Gottman, 1990,1993; Bradbury \& Fincham, 1992).

Assim como na Teoria da Troca Social essa orientação teórica não tenta explicar o mecanismo pelo qual esses julgamentos da satisfação conjugal mudam com o tempo. Em ambas as teorias existe um entendimento do momento e da interação, mas não de como esses eventos vão se modificando ao longo do tempo; o que se faz necessário para entender porque alguns casais se mantêm casados.

A despeito dessas críticas, a Teoria Comportamental se desenvolveu muito nas últimas décadas, especialmente pelos estudos de Gottman (1990;1993). São investigações que utilizam como método observar e mensurar as respostas comportamentais dos cônjuges em simulações de situações cotidianas tanto de conflito quanto prazerosas, realizadas em laboratório (Gottman \& Katz, 1989; Burleson \& Denton, 1997; Gottman \& Cols., 1998).

Essa perspectiva interacional pode ser percebida, ao mesmo tempo, como ponto forte e fraco da teoria, porque embora analise as interações dos casais, não considera o meio em que vivem. Ao contrário da Teoria da Troca Social que inclui todos os contextos, essa orientação deixa de lado variáveis como nível educacional, ou eventos estressores que, provavelmente, têm grande influência na vida conjugal (Davis, Hops, Alpert \& Sheeber, 1998).

Seguindo essa linha interacional com o foco, nas experiências prévias dos cônjuges, a Attachment Theory de Bowlby (1984) - também é bastante utilizada no entendimento das relações conjugais. Para 
o autor, as primárias estabelecidas entre a criança e seus cuidadores podem ser caracterizadas por um tipo de vínculo denominado por ele de "apego", que define um modelo interno para a criança de como são os relacionamentos íntimos entre as pessoas e poderá determinar a forma de ela se relacionar futuramente com os outros.

Teoricamente, o número de tipos de apego que podem ser estabelecidos é muito grande, mas as pesquisas realizadas por pesquisadores, depois de Bowlby (1984) ter dado início a essa temática, demonstraram três tipos mais freqüentes: o seguro, o ansiosoambivalente e o ansioso-evitativo. O apego seguro é o mais comumente observado entre as mães e os bebês, sendo visto como o ideal, em que os pais estão disponíveis aos filhos e se apresentam como base segura para as crianças explorarem novos estímulos. O tipo de apego ansioso-ambivalente descreve pais inconsistentes no cuidado com os filhos, o que gera crianças que necessitam e desejam a presença dos pais e, ao mesmo tempo, se ressentem de sua inconstância. $\mathrm{O}$ apego ansioso-evitativo classifica pais que não são responsivos e geram filhos que evitam o contato com eles.

Essa teoria foi aplicada aos relacionamentos adultos por Hazan e Shaver $(1987,1994)$ onde o tipo de apego e o modo de se relacionar estabelecido entre a criança e os cuidadores serve como parâmetro para sua compreensão na vida adult, sendo uma perspectiva teórica que enfatiza a repetição das experiências vividas na família de origem. Assim, a satisfação conjugal se relacionaria ao tipo de apego estabelecido na infância. Crianças que vivenciaram uma relação de segurança e afeto com seus pais seriam adultos que teriam mais probabilidades de repetir esse modelo em seus casamentos e, de se sentirem-se mais felizes.

Essa orientação teórica considera a importância das histórias pregressas dos cônjuges para a qualidade conjugal, o que não acontece na da Troca Social e na Comportamental. Entretanto, não é explicado pelos autores como se estabelece esse processo, uma vez que cada cônjuge tem um tipo de apego na infância e que esse não é necessariamente igual ao do parceiro. Então, como se efetiva essa relação? Casais com diferentes experiências de apego teriam mais dificuldades? E os com o mesmo tipo seriam mais satisfeitos? Esses questionamentos não são explicados pelos autores que aplicaram essa teoria à conjugalidade (Hazan \& Shaver, 1987; 1994). Ademais, nela não há espaço para o contexto em que se insere o casal, já que considera só o tipo de apego dos cônjuges e suas influências na relação deles.

Em contraponto a essa visão, a Crisis Theory, derivada do trabalho de Hill (1949), analisa como as famílias reagem às situações de crise. Por que algumas, nas adversas encaram e superam os desafios enquanto outras parecem se desorganizar? Inicialmente, o autor pensou essa teoria para famílias, mas, depois, ela foi aplicada ao entendimento da conjugalidade.

No final dos anos quarenta, Hill (1949) desenvolveu um modelo nomeado $\mathrm{ABCX}$ em que os eventos estressantes são os que requerem adaptação da família. A quantidade de recursos varia entre as famílias e as levam a diferentes níveis de reação aos eventos estressantes o que modifica o tipo de impacto para cada uma. Em qualquer circunstancia, a quantidade de recursos disponíveis para uma família enfrentar uma situação estressante vai definir a natureza da crise que poderá gerar para ela e sua capacidade de superação. A adaptação e superação são definidos pelo autor como a preservação da unidade do sistema familiar, que possibilite o desenvolvimento e crescimento de seus membros.

$\mathrm{Na}$ década de oitenta, essa teoria foi expandida por McCubbin e Patterson (1982) que adicionaram a variável tempo ao modelo, considerando que o tipo de resposta da família pode mudar ao longo do tempo e, ainda, que as respostas à crise de hoje podem levara a mais adaptativas no futuro.

Apesar de este modelo ter sido desenvolvido para explicar o funcionamento familiar, muitos pesquisadores utilizaram essa teoria para compreender e prever crises de casais. Nesta perspectiva, o declínio da satisfação conjugal e a separação seriam reflexos de incapacidades de superar crises. Em geral, casais que enfrentam mais situações estressantes podem ser mais vulneráveis a problemas conjugais se não tiverem a quantidade de recursos necessária à superação e readaptação.

Essa teoria apresenta um avanço às anteriormente descritas porque, além de considerar as influ- 
ências do contexto na qualidade conjugal, ainda focaliza os processos. Ou seja, enquanto a Teoria da Troca Social vê as variáveis do meio como fundamentais para a percepção dos cônjuges sobre sua satisfação conjugal, a da Crise além disso enfatiza a importância do processo dinâmico do relacionamento. Nesta orientação teórica, os casais são vistos como agentes ativos de seus relacionamentos na interação com o ambiente em que estão inseridos (Karney \& Bradbury,1995).

A partir desta perspectiva, a satisfação conjugal seria decorrente da capacidade do casal de superar as crises e readaptar-se a elas; da mesma forma, a estabilidade seria abalada quando houvesse falha na adaptação a algum evento estressante, o que geraria problemas aos cônjuges.

A limitação apontada à teoria refere-se à não especificação pelos autores, tanto Hill (1949) quanto McCubbin e Patterson (1982) aos tipos de respostas dos cônjuges que seriam mais adaptativas.

As quatro teorias descritas são as que mais influenciaram as pesquisas sobre qualidade conjugal, entretanto, a do Interacionismo Simbólico e a dos Sistemas Familiares também tiveram um papel importante, embora não tão significativo (Musitu \& Cava, 2001).

A Teoria do Interacionismo Simbólico, assim como a da Crise, foram aplicadas primeiramente ao entendimento de famílias, mas suas contribuições têm sido utilizadas para a compreensão dos fenômenos conjugais.

Especificamente a do Interacionismo Simbólico tem como conceito principal que a família é um grupo de pessoas em interação que criam seus símbolos e seus significados e esses vão influenciar na formação da identidade dos membros, na transmissão dos valores familiares e aprendizagem dos papéis sociais que cada um deve desempenhar (Gracia \& Musitu, 2000).

A partir desta perspectiva teórica, Burr, Hill, Nye e Reiss (1979) desenvolveu-se um modelo de funcionamento familiar. Para o autor, cada indivíduo possui uma representação dos papéis sociais que desempenha, por exemplo, de esposo, de filho. Baseado nesses papéis dos membros da família, ele admite que a avaliação de cada um sobre quão ajustado está à idéia que tem sobre seu papel, gera maior ou menor nível de satisfação no funcionamento de todo núcleo familiar. Assim, a qualidade conjugal seria resultado da avaliação que cada cônjuge faz de sua adequação ao esperado do papel de esposo ou esposa. Então, um homem se vê desempenhando bem a representação que tem do que é um esposo, estará satisfeito em sua relação conjugal.

Neste entendimento teórico percebe-se, como na Teoria da Troca Social e na da Crise, a importância do contexto em que os sujeitos envolvidos se inserem e da auto-avaliação para a qualidade conjugal. Aí, o nível de satisfação do sujeito vai depender da sua auto-avaliação sobre seu papel de esposo (a) e o quão adequado pensa ele estar no seu contexto.

A Teoria dos Sistemas Familiares, embora seja a base da da Crise, tem no modelo de Olson, Sprenkle e Russel (1979) sobre funcionamento familiar uma representação extremamente significativa, já que gerou um grande número de pesquisas.

Olson (2000) desenvolveu o modelo circumplexo dos sistemas conjugal e familiar. Nele, o nível de funcionalidade do casal vai variar em função de três dimensões: a coesão, a adaptabilidade e a comunicação.

A coesão é definida como o vínculo emocional que os membros da família têm em relação um ao outro. Há quatro níveis de coesão, identificados no quadro abaixo:

Quadro 2. Modelo Circunplexo de Olson (2000), dimensão coesão:

\begin{tabular}{|l|l|}
\hline \multicolumn{1}{|c|}{ Tipo de Relação } & \multicolumn{1}{c|}{ Nível de Coesão } \\
\hline Emaranhado & Muito Alto \\
\hline Conectado & Moderado a Alto \\
\hline Separado & Moderado a Baixo \\
\hline Desprendido & Muito Baixo \\
\hline
\end{tabular}

A hipótese do modelo é que níveis centrais ou equilibrados de coesão (separado e conectado) geram funcionamento ótimo familiar e conjugal. Os extremos ou desequilibrados (desprendido e emaranhado) são normalmente relacionados a problemas a longo prazo para os relacionamentos.

A segunda dimensão é a adaptabilidade familiar entendida como o potencial de mudança/adapta- 
ção na liderança, papéis e regras do sistema. A tabela abaixo traz os quatro níveis de adaptabilidade:

Quadro 3. Modelo Circunplexo de Olson (2000), dimensão adaptabilidade:

\begin{tabular}{|l|l|}
\hline Cipo de Relaça & Nivel de Adaptabilidade \\
\hline Caótico & Muito Alto \\
\hline Flexível & Moderado a Alto \\
\hline Estruturado & Moderado a Baixo \\
\hline Rígido & Muito Baixo \\
\hline
\end{tabular}

Assim como na coesão, a hipótese é que os níveis equilibrados (estruturado e flexível) conduzem a um melhor funcionamento familiar e conjugal, sendo os extremos (rígido e caótico) os mais problemáticos para as famílias e casais.

A comunicação é uma dimensão vista como facilitadora e fundamental na promoção de movimentos nas outras duas. Por ser facilitadora ela não é mensurada como a coesão e adaptabilidade e para o autor estaria inserida nelas.

Ao analisar o modelo de Olson percebe-se a semelhança com a Teoria da Crise pelo enfoque na capacidade da adaptação das famílias e casais frente aos desafios do meio. Ambas consideram fundamental para o nível de satisfação conjugal que os casais possam ter recursos para enfrentar os problemas e se adaptarem às dificuldades.

Neste sentido, pode-se perceber que das teorias sobre a qualidade conjugal expostas, quatro delas (Teoria da Troca Social, Teoria da Crise, Interacionismo Simbólico e Teoria dos Sistemas Familiares) se assemelham ao considerar a influência do contexto, da auto - avaliação e da capacidade de superar problemas como variáveis essenciais na definição da qualidade conjugal.

Nesta perspectiva, Karney e Bradbury (1995) desenvolveram um modelo integrando essas teorias e os achados de 115 pesquisas longitudinais realizadas sobre qualidade e estabilidade conjugal nas últimas cinco décadas. O modelo denominado Vulnerability Stress Adaptation é a integração das Teorias do Apego, da Crise e Comportamental.

O modelo sustenta que os casais necessitam se adaptar a uma grande variedade de eventos estressantes e circunstâncias que surgem no curso da vida a dois. A capacidade do casal de se adaptar a esses acontecimentos vai depender do nível de estresse que eles experimentam e das características prévias que cada um traz ao casamento. A acumulação destas experiências vividas nos processos adaptativos influencia a percepção da sua qualidade conjugal, o que, por sua vez, atua na estabilidade do casamento.

O modelo salienta a importância de três grupos de variáveis que definem a qualidade conjugal. $\mathrm{O}$ contexto, os recursos pessoais dos cônjuges e os processos adaptativos. Esses grupos de variáveis se influenciam mutuamente e devem ser entendidos de forma integrada.

O contexto em que os casais estão inseridos e as conseqüentes situações estressantes geradas pelo mesmo são fundamentais na definição de qualidade que experienciam. Pesquisas mostram que os expostos a situações estressantes como doenças, desemprego, problemas familiares relatam níveis mais baixos de satisfação conjugal (Belsky,1984).

Da mesma forma, os recursos pessoais de cada cônjuge são fundamentais na interação com as outras variáveis e o consequiente nível de qualidade conjugal. Dados de pesquisas mostram que as experiências na família de origem respondem por $10 \%$ da qualidade conjugal dos cônjuges (Falcke, 2003). Ademais, o nível educacional também é apontado como importante nesse processo (Griffin,1993) assim como as características de personalidade (Ye \& cols., 1999).

O último grupo de variáveis responde aos processos adaptativos e se refere à capacidade dos casais em superar os desafios gerados pelo contexto em que estão inseridos e sua conseqüente adaptação aos mesmos. Pesquisas mostram que as dificuldades sempre existem em um casamento e que a diferença na qualidade conjugal está na forma como os cônjuges irão resolver esses problemas (Bradbury \& cols., 2000).

Atualmente é aceito que casais possam discordar e entrar em conflito e ainda assim apresentar altos níveis de satisfação, dependendo da maneira de resolver esses conflitos. Em última análise, a qualidade conjugal vem como resultado desse processo interativo entre o contexto, os recursos pessoais dos 


\section{Clarisse Mosmann}

cônjuges e os processos adaptativos (Webster-Stratton \& Hammond, 1999).

Ao se analisarem definições do conceito de qualidade conjugal através das sete teorias expostas percebe-se que elas se assemelham ao considerarem algumas variáveis como fundamentais na sua definição. Embora seja bastante complexa a diferenciação conceitual entre as teorias, o quadro a seguir mostra as variáveis tidas como fundamentais na definição da qualidade conjugal comparativamente:

Quadro 4: Quadro conceitual e diferencial das variáveis essenciais na definição da qualidade conjugal

\begin{tabular}{|c|c|}
\hline Teoria & Variáveis Essenciais \\
\hline $\begin{array}{l}\text { Teoria Comportamental } \\
\text { (Gottman,1982). }\end{array}$ & \multirow{2}{*}{ RECURSOS PESSOAIS } \\
\hline $\begin{array}{l}\text { Teoria do Apego } \\
\text { (Bowlby ,1984). }\end{array}$ & \\
\hline $\begin{array}{l}\text { Teoria da Troca Social } \\
\text { (Levinger, 1965,1976). }\end{array}$ & \multirow[t]{2}{*}{ CONTEXTO } \\
\hline $\begin{array}{l}\text { Teoria da Crise } \\
(\text { Hill, 1949). }\end{array}$ & \\
\hline $\begin{array}{l}\text { Teoria do Interacionismo } \\
\text { Simbólico } \\
\text { (Burr \& cols., 1979). }\end{array}$ & AUTO-AVALIAÇÃO \\
\hline $\begin{array}{l}\text { Teoria dos Sistemas } \\
\text { Familiares } \\
\text { (Olson \& cols., 1979, 2000). }\end{array}$ & ADAPTABILIDADE \\
\hline Modelo de Adaptação da & CONTEXTO \\
\hline Vulnerabilidade ao Estresse & RECURSOS PESSOAIS \\
\hline (Karney \& Bradbury,1995). & PROCESSOS ADAPTATIVOS \\
\hline
\end{tabular}

Pode-se identificar no quadro acima que o Modelo de Adaptação da Vulnerabilidade ao Estresse (Karney \& Bradbury,1995), integra as teorias ao considerar como determinantes da qualidade conjugal o contexto, os recursos pessoais e os processos adaptativos.

A partir desse conceito identifica-se que a qualidade conjugal é multidimensional. A satisfação ou felicidade é uma dimensão dele e seria semelhante teoricamente ao conceito de ajustamento que vê no ajustamento conjugal um processo em que a satisfação está incluída.

Apesar de não haver um consenso sobre essa questão Johnson, White, Edwards e Booth (1986) comprovaram empiricamente que a satisfação e a qualidade conjugal são dimensões distintas e que a utilização de mais de uma variável para descrever esta úl- tima permite maior entendimento do fenômeno. Da mesma forma, a aceitação de que na atualidade a tendência das pesquisas é fazer um entendimento dos processos conjugais e para isso a adoção de conceitos multidimensionais se faz não só necessária como fundamental.

\section{Considerações finais}

Retomando o objetivo inicial do presente trabalho de mapear o conceito de qualidade conjugal, após revisar a literatura da área, identifica-se que o mesmo não pode ser definido simplesmente pela avaliação dos cônjuges sobre seu matrimônio; ela é resultado de um processo dinâmico e interativo do casal que resulta na avaliação que cada cônjuge tem do nível de qualidade que experimenta em sua união.

O conceito é vulnerável a todas as variáveis que compõem sua definição. Mais especificamente, o contexto, os recursos pessoais dos cônjuges, e os processos adaptativos aparecem como as três grandes dimensões que levariam à auto-percepção e a um conseqüente nível de qualidade conjugal.

Essas três dimensões reúnem um número infinito de variáveis tais como as experiências na família de origem, o nível educacional, as características de personalidade, a fase do ciclo vital em que se encontra o casal, dentre outras.

Estas são variáveis difíceis de serem hierarquizadas, pois não há como determinar a maior importância de umas em relação a outras. O que ocorre no estudo das três dimensões é uma escolha, por parte dos pesquisadores, de algumas variáveis na busca de entender de forma mais específica uma parte deste constructo.

Por esse motivo, as pesquisas longitudinais seriam as mais indicadas para obter dados próximos à realidade dinâmica implícita no conceito de qualidade conjugal. Entretanto, tais pesquisas requerem um grande investimento financeiro e de tempo. Assim, estudos de corte transversal podem auxiliar na compreensão do fenômeno se buscarem avaliar, por meio das metodologias empregadas, mais de uma variável relacionada às três dimensões (contexto, recursos pessoais dos cônjuges e os processos adaptativos).

Independentemente da opção metodológica, é fundamental, para o desenvolvimento das pesquisas 
na área, o entendimento de que a qualidade conjugal é multidimensional e que os estudos devem centralizar seus esforços na busca do mapeamento das relações entre as variáveis sem o objetivo de estabelecer relações de causa e efeito.

\section{Referências}

Amato, P. R., Johnson, D. R., Booth, A., \& Rogers, S. J. (2003). Continuity and change in marital quality between 1980 and 2000. Journal of Marriage and Family, 65, 1-22.

Beach, S. R. H; Katz, J.; Sooyeon, K., \& Brody, G. (2003). Perspective effects of marital satisfaction on depressive symptoms in established marriages: A dyadic model. Journal of Social and Personal Relationships, 20, 355-371.

Belsky, J. (1984). The determinants of parenting: a process model. Child Development, 55, 83-96.

Bowlby, J. (1984). Apego e Perda. São Paulo: Martins Fontes.

Bradbury, T. N., \& Fincham, F. D. (1992). Attributions and behavior in marital interaction. Journal of Personality and Social Psychology, 63, 613-628.

Bradbury, T. N., Fincham, F. D., \& Beach, S.R.H. (2000). Research on the nature and determinants of marital satisfaction: a decade in review. Journal of Marriage and the Family, 62, 964-980.

Bronfenbrenner, U. (1996). A ecologia do desenvolvimento humano: experimentos naturais e planejados. Porto Alegre: Artes Médicas.

Burleson, B. R., \& Denton, W. H. (1997). The relationship between communication skill and marital satisfaction: some moderating effects. Journal of Marriage and Family, 59, 884-902.

Burman, B., \& Margolin, G. (1992). Analysis of association between marital relationships and health problems: An interactional perspective. Psychological Bulletin, 112, 39-63.

Burr, W. R., Hill, R., Nye, F. I., \& Reiss, I. (1979). Contemporary theories about the family. New York: Free Press.
Cotton, S. R.; Burton, R. P. D., \& Rushing, B. (2003). The mediating effects of attachment to social structure and psychosocial resources on the relationship between marital quality and psychological distress. Journal of Family Issues, 24, 547-577.

Davis, B. T., Hops, H., Alpert, A., \& Sheeber, L. (1998). Child responses to parental conflict and their effect on adjustment: a study of triadic relations. Journal of Family Psychology, 12,163-177.

Erel, O., \& Burman, B. (1995). Interrelatedness of Marital Relations and Parent-Child Relations: A Meta-Analytic Review. Pshychological Bulletin, 118, 108-132.

Falcke, D. (2003). Águas passadas não movem moinhos? Tese de Doutorado. Pontifícia Universidade Católica do Rio Grande do Sul, Porto Alegre.

Falcke, D., Diehl, J. A., \& Wagner, A. (2002). Satisfação Conjugal na Atualidade. Em A. Wagner (Org.), Família em Cena. (pp.172-188). Petrópolis: Vozes.

Féres-Carneiro, T. (2003). Separação: o doloroso processo de dissolução da conjugalidade. Estudos de Psicologia, 8, 367-374.

Fincham, F. D., \& Bradbury, T. N. (1987). The Assessment of Marital Quality: A Reevaluation. Journal of Marriage and the Family, 49, 797-809.

Glenn, N. D. (1998). The Course of Marital Success and Failure in Five American 10- Year Marriage Cohorts. Journal of Marriage and the Family, 60, 569-576.

Gottman, J. M. (1982). Temporal form: Toward a new language for describing relationships. Journal of Marriage and the Family, 44, 943-962.

Gottman, J. M. (1990). How marriages change. In G. R. Patterson (Ed.), Depression and aggression in family interaction (pp.75-101). Hillsdale, N.J.: Erlbaum.

Gottman, J. M. (1993). A theory of marital dissolution and stability. Journal of Family Psychology, 7, 57-75.

Gottman, J. M., \& Katz, L. F. (1989). Effects of Marital Discord on Young Children's Peer Interaction and Health. Developmental Psychology, 25, 373-381. 
Gottman, J. M., \& Notarius, C. I. (2002). Marital Research in the $20^{\text {th }}$ Century and a Research Agenda for the $21^{\text {st }}$ Century. Family Process, 41, 159-197.

Gottman, J. M.; Coan, J.; Carrere, S., \& Swanson, C. (1998). Journal of the Marriage and the Family, 60, 5-22.

Gracia, E., \& Musitu, G. (2000). Psicologia Social de la Familia. Barcelona: Paidós.

Griffin, W. A. (1993). Transitions from negative affect during marital interaction: husband and wife differences. Journal of Family Psychology, 6, 230244.

Hazan, C., \& Shaver, P. R. (1987). Romantic love conceptualized as an attachment process. Journal of Personality and Social Psychology, 52, 511-524.

Hazan, C., \& Shaver, P. R. (1994). Attachment as an organizational framework for research on close relationships. Psychological Inquiry, 5, 1-22.

Heyman, R., Sayers, A., \& Bellack, S. (1994). Global marital satisfaction versus marital adjustment: an empirical comparison of three measures. Journal of Family Psychology, 8, 32-446.

Hill, R. (1949). Families under stress. New York: Harper.

IBGE (2003). Anuário Estatístico Brasileiro. Fundação Instituto Brasileiro de Geografia e Estatística. Rio de Janeiro, v.23.

Jablonski, B. (1991). Até que a vida nos separe: a crise do casamento contemporâneo. Rio de Janeiro: Agir.

Johnson, D. R., Amoloza, T. O., \& Booth, A. (1992). Stability and Developmental Change in Marital Quality: A Three-Wave Panel Analysis. Journal of the Marriage and the Family, 54, 582594.

Johnson, D. R., White, L. K., Edwards, J. N., \& Booth, A. (1986). Dimensions of marital quality: Toward methodological and conceptual refinement. Journal of Family Issues, 7, 31-49.

Karney, B. R., \& Bradbury, T. N. (1995). Assessing longitudinal change in marriage: An introduction to the analysis of growth curves. Journal of Marriage and the family, 57, 1091-1108.
Kline, S. L., \& Stafford, L. (2004). A comparison of interaction rules and interaction frequency in relationship to marital quality. Communication Reports, 17 , 11-26.

Levinger, G. (1965). Marital cohesiveness and dissolution: An intregrative review. Journal of Marriage and the family, 27, 19-28.

Levinger, G. (1976). A social psychological perspective on marital dissolution. Journal of Social Issues, 32, 21-47.

Lewis, R. A., \& Spanier, G. B. (1979). Theorizing about the Quality and Stability of Marriage. In W. R. Burr, R. Hill, F. I. Nye, \& I. L. Reiss (Ed.), Contemporary Theories about The Family (pp. 268-294). New York: The Free Press.

Lewis, R. A., \& Spanier, G. B. (1982). Marital Quality, Marital Stability and Social Exchange. In F. I. Nye (Eds.), Family Relationships, Rewards and Costs. (pp. 49-65). Beverly Hills: Sage Publications.

McCubbin, H. I., \& Patterson, J. M. (1982). Family Adaptation to Crises. In H. I. McCubbin, A. E. Cauble, \& J. M. Patterson (Eds.), Family Stress, Coping and Social Support (pp.26-47). Springfield, Il: Charles C. Thomas.

Musitu, G., \& Cava, M. J. (2001). La Familia y la Educación. Barcelona: Octaedro.

Neff, L. A., \& Karney, B. R. (2003). The Dynamic Structure of Relationship Perceptions: Differential importance as a strategy of relationship maintenance. Personality and Social Psychology Bulletin, 29, 1433-1446.

Norton, R. (1983). Measuring Marital Quality: A critical look at the dependent variable. Journal of Marriage and the Family, 45, 141-151.

O’Leary, K. D., \& Smith, D. A. (1991). Marital Interactions. Annual Review of Psychology, 42, 191212.

Olson, D. (2000). Circumplex Model of Marital and Family Systems. Journal of Family Therapy, 22, 144-167.

Olson, D., Sprenkle, D. H., \& Russel, C. S. (1979). Circumplex model of marital and family systems: I- Cohesion and adaptability dimensions, family types and clinical applications. Family Process, 18, 3-28. 
Perry-Jenkins, M., Repetti, R. L., \& Crouter, A. C. (2000). Work and Family in the 1990s. Journal of Marriage and the Family, 62, 981-998.

Shek, D. T. (1998). Linkage between Marital Quality And Parent-Child Relationship. Journal of Family Issues, 19, 687-704.

Shek, D.T. (2001). Paternal and Maternal Influences on Family Functioning Among Hong Kong Chinese Families. The Journal of Genetic Psychology, 162, 56-74.

Spanier, G. B., \& Cole, C. L. (1976). Toward clarification and investigation of marital adjustment. International Journal Sociology of the Family, 6, 121-146.

Thibaut, J. W., \& Kelley, H. H. (1959). The social psychology of groups. New York: Wiley.

Uebelacker, L. A.; Courtnage, E. S., \& Whisman, M.A. (2003). Correlates of depression and marital dissatisfaction: Perceptions of marital communication style. Journal of Social and Personal Relationships, 6, 757-769.

Webster-Stratton, C., \& Hammond, M. (1999). Marital conflict management skills, parenting style, and early - onset conduct problems: processes and pathways. Journal of Child Psychology and Psychiatry, 40, 917-927.

Whisman, M. A. (1999). Marital dissatisfaction and psychiatric disorders: Results from the National Comorbidity Survey. Journal of Abnormal Psychology, 108, 701-706.

Ye, M.; Wen, S., \& Wang, L. (1999). Quality of marriage and personality match of couples. Chinese Mental Health Journal, 13, 298-299.

Artigo derivado do ensaio temático apresentado no exame de qualificação desenvolvido pela autora principal no doutorado em Psicologia Social e da Personalidade, PUCRS. 